\section{Genentech prospects gut and soil}

Genentech has entered into two new deals to investigate bacteria as a source of new drugs, targets, biomarkers and live-bacteria therapeutic products. With Cambridge, UK-based Microbiotica, Genentech will pay up to $\$ 534$ million, including milestones to scan human gut microbiome signatures from patients taking part in clinical trials with etrolizumab and IL22-Fc for ulcerative colitis and Crohn's disease. Microbiotica, which was spun out of the Wellcome Sanger Institute in 2016, uses a platform that combines a microbiome Culture Collection with a Reference Genome Database that allows bacterial identification in patient samples. The resulting datasets are analyzed by Al techniques to identify microbiome patterns linked to phenotype. The pathogenesis of inflammatory bowel disorders is linked to disruptions in the gut microbiome, but the majority of these microbes have yet to be studied. The partners will also use Microbiotica's physical Culture Collection to evaluate bacteria in translational models, including humanized microbiome mouse models. "We believe the microbiome represents a new paradigm in biomedicine, both for understanding drug response and as a novel therapeutic modality," said Genentech in a statement. The second Genentech deal is with Lodo Therapeutics, which will receive up to $\$ 969$ million, including milestone payments to search for new drugs derived from the rich sources of microbial DNA in soil (targets and diseases were not disclosed). Lodo, which was launched out of Rockefeller University in 2016, collects soil samples from across the US, from which microbial DNA is extracted. The DNA is then cloned into easily cultured bacterial hosts, allowing for the construction of large DNA libraries that are a potential source of natural products. For example, Lodo researchers identified a new family of antibiotics that killed bacteria resistant to vancomycin and methicillin-resistant Staphylococcus aureus (Nat. Microbiol. 3, 415-422, 2018).

\footnotetext{
6 "Social scientists would read this and say it is really elementary, why do they bother, but [understanding of these concepts is] not typical for researchers working on a drug or device." Sharon Terry, CEO of Genetic Alliance, counters criticism of the first of four FDA guidances on patientfocused drug development, which some found to be basic, lacking in practical detail. (BioCentury, 12 July 2018)

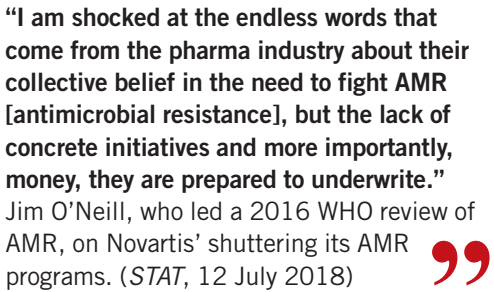

"I am shocked at the endless words that come from the pharma industry about their collective belief in the need to fight AMR [antimicrobial resistance], but the lack of concrete initiatives and more importantly, money, they are prepared to underwrite." Jim O'Neill, who led a 2016 WHO review of programs. (STAT, 12 July 2018)
}

decision independent of clinical input from an eye specialist, providing a complete diagnostic workflow that primary care offices can implement, not just specialized ophthalmology clinics and practices. The company spent seven years working with FDA to develop the best approach to demonstrate the software's safety and effectiveness, as the higher level of risk demanded additional scrutiny by the agency. "That is what we need to drive down the price and improve the quality of healthcare," says founder and president Michael Abramoff. The FDA cleared IDx DR in April.

Retinal camera operators generally are highly trained, but the IDx DR system, which also requires a designated robotic retinal camera, needs only three to four hours of operator training for use. The AI directs which part of the eye to image and ensures the images are of good quality. "It's an interactive process with the operator being guided by the AI to take the images," says Abramoff. The AI then analyzes the images for diagnosis. "It's much more intricate than uploading images and getting a result. It depends on the quality of the operator and the images, and that is what FDA wanted us to validate," he says. Also, unlike the radiology companies, IDx conducted a prospective study, which is different than using previously collected data in a retrospective validation trial.

"Everybody thinks radiology is the field out in front," adds Topol. But the more rigorous studies, he says, have come out of ophthalmology. Topol recently became an advisor to Verily Life Sciences, which is teaming up with the Google AI in Healthcare group to develop technology for the earlier detection of diabetic retinopathy and diabetic macular edema.

Retinal scans could potentially offer up a wealth of biomarkers on which to base a broad health workup. Because the eye is chock full of arteries and neurons, clinical researchers have hypothesized that they could be used to detect cardiovascular or neurodegenerative disorders. "Retinal imaging is the window into the body of the eye and could wind up showing how well blood pressure or diabetes is being controlled," Topol says, or even predict early signs of dementia. Individuals eventually will be able to take the measurements using a smartphone, without having to see a physician. "This is going to be a consumer story someday," he says.

As with stroke, early detection of diabetic retinopathy is important, as most patients do not have symptoms until the path to irreversible vision loss and blindness is already set. When caught early, about $95 \%$ of these patients can be treated effectively. Plus, says Abramoff, "We know the exact biomarkers to look for, like hemorrhages, micro-aneurysms and exudates (cells and fluid that have leaked out from blood vessels). That makes it possible to develop the AI algorithms."

To develop IDx DR, it was important to know how the neural network made associations between disease and specific image features, Abramoff says. But often when algorithms are used successfully in deep learning, what goes on in multiple convoluted layers is hidden from the algorithm designers. It's a black box. One recent example of a black box algorithm is a study from the University of Heidelberg on melanoma identification using Google's convolutional neural network (CNN). The CNN outperformed an international group of dermatologists, the majority of whom are experts in the field. "The CNN learns by looking at more than 100,000 lesions labeled as either benign or malignant. It will create its own "pixel-based" rules for the correct diagnosis," says study first-author Holger Haenssle. "We cannot exactly explain the rules the CNN makes up."

Black box algorithms become an interesting dilemma for regulators: if it works well and is validated, why worry about the fact that the mystery is not understood? That may not be the feeling in Europe following enactment of the European Union's General Data Protection Regulation (GDPR) in May 2018. The GDPR implies "right to explain" language, possibly meaning an algorithm may not be used without unravelling its black box.

The FDA has set no such bar in the US. Imagen, Viz.ai and IDx have all taken advantage of the FDA's de novo premarket review pathway, which allows for marketing authorization for some new types of low- to moderate-risk medical devices. "You are seeing FDA not just approving these tools, they are accelerating them," says Eric Perakslis, CSO at Datavant and a former chief information officer at FDA.

This all dovetails with the agency's goals of spurring innovation. If the software on a device is part of a clinical workflow, rather than diagnosing or treating something, FDA may fasttrack it through a precertification program. Unlike in the European Union, the US agency is "decreasing supervision of lower benefit/ lower risk tasks," Perakslis says.

Many different specialties are on the AI path. Radiology and ophthalmology are the two leading ones, with pathology and dermatology close behind, along with cardiology, gastroenterology (e.g., for interpretation of the video images during colonoscopy), mental health support for psychologists and psychiatrists, even tools for paramedics and pharmacists, Topol says, adding that "no specialty I know of is spared from AI effects."

Mark Ratner Tequesta, Florida 